Acta Hispanica (2020) Supplementum II: 721-736

\title{
CUIDADO DEL AGUA EN CLAVE DEL PENSAMIENTO AMBIENTAL COMPLEJO-SUR
}

\author{
NATALia SÁNCHEZ GOMEZ
}

Universidad de Boyacá

\begin{abstract}
Resumen: El agua no solo constituye un pilar fundamental en las dinámicas productivas y sociales de un territorio, representa un bien común que posibilita la vida. El uso compartido de este elemento genera una serie de impactos, interdependencias e incertidumbres en los procesos de gestión y toma de decisiones. Reconociendo que la participación de diversos actores y la integración de los conocimientos locales son claves en la construcción de un entendimiento de los sistemas socioecológicos y de sus posibles respuestas a los cambios, la presente ponencia, acoge la idea de que la gestión del agua debe repensarse en clave de un Pensamiento Ambiental que apueste a otras formas de conocer la realidad y por lo tanto de transformarla, planteando la urgencia de una pedagogía fundada en un pensamiento que desde las culturas de la América - Abya- Yala, propicien el cuidado como camino hacia un habitar poético-sur. Las reflexiones que se comparten en este trabajo, surgen a partir de la etapa inicial de la tesis doctoral "Praxis pedagógica en clave de pensamiento ambiental complejo sur" la cual se desarrolla en el marco del proyecto de investigación denominado "Resiliencia y Gestión de Sistemas Socio-Ecológicos Estratégicos para la Actividad Agrícola, en Escenarios de Variabilidad Climática. Caso de Estudio: Lago Sochagota (Boyacá, Colombia)", en donde a partir del diálogo interdisciplinarios e intercultural, se ha logrado identificar obstáculos epistemológicos que dificultan una gestión orientada al cuidado del agua.
\end{abstract}

Palabras clave: gestión ambiental, cuidado, pensamiento ambiental, diálogo de saberes.

Abstract: Water is not only a fundamental pillar in the productive and social dynamics of a territory, it represents a common good that makes life possible. The shared use of this element generates a series of impacts, interdependencies and uncertainties in the processes of management and decision making. Recognizing that the participation of diverse actors and the integration of local knowledge are key in the construction of an understanding of the socio-ecological systems and their possible responses to changes, this paper, welcomes the idea that water management must rethink in the key of an Environmental Thought that bets to other ways of knowing the reality and therefore of transforming it, raising the urgency of a pedagogy founded on a thought that from the cultures of the America - Abya -Yala, propicien the care as path towards a poetic-south dwelling. The reflections that are shared in this work, arise from the initial stage of the doctoral thesis "Pedagogic Praxis in the key of complex southern environmental thinking" which is developed within the framework of the research project called "Resilience and Management of Socio-Ecological Strategies Systems for Agricultural Activity, in Scenarios of Climate Variability. Case Study: Sochagota Lake (Boyacá, Colombia)", where from the interdisciplinary and intercultural dialogue, it has been possible to identify the epistemological obstacles for a management oriented to the care of water.

Keywords: Environmental Management, Care, Thinking Environmental, Dialogue of Knowledge.

Acta Hispanica, Hungría, Supplementum II: 721-736, 2020, ISSN: 1416-7263 |721 
Cuidado del agua en clave del pensamiento ambiental complejo-sur

\section{Entretejido de sentipensares sobre el Agua como bien común}

\subsection{El Agua, elemento indispensable para la vida}

Aunque estas letras se teclean en un aquí y un ahora, están impregnadas de otros tiempos, otros lugares, habitados por otras voces. En coro con esas voces, este ensayo se escribe en tercera persona, permitiendo el uso del "nosotros" en algunas ideas en donde se considera necesario evidenciar un sentir colectivo sobre aquello que "nos une" no solo como especie humana sino como seres vivos en un planeta vivo.

Luego de intentar incesantemente distinguir la humanidad del resto de animales, ha llegado el momento de preguntar sobre lo que une al ser humano con el resto de los seres vivos, desde la física cuántica se diría que la energía, desde la sociología, la cultura, desde la ingeniería, la tecnología, desde la biología, el ADN, desde la economía, la estupidez humana, y así se podría continuar con tantas respuestas como personas en el mundo. Para este escrito centraremos la atención en aquello que nos une y a la vez nos desune, el Agua, sí, con mayúscula, el Agua con nombre propio y no el agua como objeto ni recurso, porque, aunque esto parezca algo trivial, para este ensayo no lo es. Aquí, el uso de las palabras no se limita a la simple transmisión de ideas, sino que cada una de ellas connota un sentido y una forma de comprender el mundo, por esto se considera que la transformación del lenguaje es un primer paso para la transformación individual y colectiva de las formas que tenemos de habitar.

Para iniciar con la discusión de este primer apartado, el cual pretende problematizar los vínculos entre el ser humano y el agua, se desarrolla un entretejido problemático que se teje desde las causas del descuido del agua como elemento de vida y no como suele hacerse, desde sus consecuencias. Esta reflexión considera que ya existe suficiente documentación científica y académica que evidencia las condiciones de contaminación de fuentes de Agua y la escasez de este elemento. Según el Informe Mundial sobre el Desarrollo de los Recursos Hídricos de las Naciones unidas publicado en el 2019, alrededor de 2.000 millones de personas sufren una fuerte escasez de agua durante todo el año y más del $90 \%$ de países en el mundo no logran brindar una cobertura de agua potable a toda su población (WWAP, 2009), información que si bien es cierto es importante para demostrar la necesidad de repensar la "gestión del agua" en el mundo, es insuficiente para comprender el problema complejo alrededor de este preciado elemento.

Ante lo anterior, la presente reflexión se orienta hacia las causas del problema del agua, considerando que permite acercarse de mejor manera a los intereses pedagógicos de este trabajo, reconociendo que la gestión del agua no debe responder únicamente a una cuestión de contaminación o escasez de un "recursos natural" sino que debe comprender el problema del agua como algo que abarca la totalidad de la vida (Maya, 1996). Desde esta perspectiva se reflexiona críticamente lo que se conoce comúnmente como "gestión ambiental", para acercarse a maneras otras de cuidado que permitan afrontar los dilemas presenten en el manejo de un bien común como el agua. 


\subsection{Repensando la gestión del Agua}

Nótese, el uso de las comillas en las palabras de gestión ambiental en el apartado anterior, esto no es algo desprevenido, al contrario, tiene una intención especifica de denotar un acento de ironía acompañado del ridículo ademán de dibujar con los dedos unas comillas en el aire, para expresar cierta duda o resquemor en el uso de la palabra. Se hace esta aclaración porque se busca que el lector de esta ponencia, interprete el uso de este tipo de comillas en este escrito, como una forma de problematizar conceptos que parecieran ser indiscutibles como "desarrollo sostenible" y "educación ambiental", pero que desde el pensamiento ambiental latinoamericano se están cuestionando con miras a develar intereses que no correspondan al bien común.

Para comprender de mejor manera lo anterior, fijemos la atención en la palabra "gestión" para ir decantando el problema que se busca exponer aquí. Antes de iniciar con esto, vale la pena aclarar que no es de interés de este trabajo desarrollar una conceptualización o teorización de lo que se entiende por gestión, simplemente se tomará una definición básica que permiten discutir algunos planteamientos que se consideran necesarios en este escrito.

En el diccionario de la Real Academia española en línea, gestión se define como «Acción o trámite que, junto con otros, se lleva a cabo para conseguir o resolver una cosa» $\mathrm{O}$ «Conjunto de operaciones que se realizan para dirigir y administrar un negocio o una empresa» (RAE, 2001), hasta aquí la gestión parte de la idea de ejecutar acciones, lo cual es algo necesario para solucionar problemas y alcanzar objetivos, pero las preguntas que se suscitan de esta lectura son ¿Qué tipo de problemas se quieren solucionar con la gestión?, ¿Qué tipo de objetivos se quieren alcanzar?. En la literatura del agua se encuentra principalmente preocupaciones relacionadas con el desarrollo de un territorio y los procesos productivos que allí se dinamizan (Apipalakul - Wirojangud - Ngang, 2015). Se hace énfasis también en los intereses de los sectores industrial, turístico, agrícola, pecuario, piscícola, de generación eléctrica, entre otros, y los conflictos que emergen del uso compartido de este "recurso" en contextos locales y regionales, presentándose un alto número interdependencias e incertidumbres en los procesos de gestión y toma de decisiones (Thompson - Owen - Lindsay - Leonard - Cronin, 2017).

Desde estas perspectivas, la gestión del Agua se preocupa por problemas de eficiencia, productividad, escasez, contaminación y se plantean objetivos de "desarrollo" técnicocientífico y tecnológico, que la mayoría de las veces son propuestos desde una visión gerencial. Con esto se quiere discutir si realmente la gestión, permite abordar el problema del agua desde su complejidad, o tan solo permite reaccionar bajo escenarios de desarrollo local de manera instrumental y sobre todo bajo la creencia de un ser humano que todo lo puede controlar y dominar.

No se trata aquí de descalificar todos los procesos de gestión del agua que se adelantan en los territorios, existen experiencias de gestión comunitaria y participativa que han logrado superar las barreras epistemológicas de una gestión mercantil del agua, sin 
Cuidado del agua en clave del pensamiento ambiental complejo-sur

embargo la apuesta de este escrito es evidenciar que existen diversas manera de relacionarse con el agua a través de otras lógicas no administrativas, desde las cuales se busca una toma de decisiones con respecto al uso y manejo del agua basadas no solo en información técnico-científica sino que desde un vínculo afectivo, espiritual y sagrado de lo vivo con el Agua.

\subsection{Lo común, lo comúnmente descuidado}

Como ya se dijo anteriormente, en este ensayo se considera que la transformación del lenguaje es un primer paso para esa tarea de construir, de-construir y re-construir los vínculos con el agua, por lo tanto superar el lenguaje dominante en el estudio de lo ambiental, que ha convertido el Agua en recurso, los ecosistemas en servicios ambientales y el ambiente en exterioridad, es una tarea que debe hacerse, si realmente se quiere comprender lo ambiental desde un pensamiento comprometido con la vida. Es así, como desde el pensamiento ambiental latinoamericano, lo ambiental es poesía, es decir poiesis comprendida como acto creador donde la ética y la estética son fundamentales para transitar nuevos caminos hacia el re-encuentro con el Agua como elemento vinculante, como un bien común.

Según Ostrom (2009), se llama los «commons» a los bienes que un grupo, comunidad o sociedad utiliza en común, los comunes o los bienes comunes como también son llamados para darle más claridad al término en español, no pueden ser objeto de mercado porque no son "propiedad" de la humanidad como muchas veces se ha planteado en la literatura antropocentrista, sino que representan el fundamento de todo tipo de vida en el planeta, especialmente el Agua que es un bien natural, universal, insustituible y vital.

Como se expresa en el manifiesto por la vida que se presentó como resultado del Simposio sobre ética y Desarrollo Sustentable, celebrado en Bogotá, Colombia, los días 2-4 de mayo de 2002 (Tangencial, 2002), los bienes comunes han quedado sujetos a las formas de propiedad, en donde confluyen múltiples intereses y por ende conflictos entre lo propio y lo ajeno; lo público y lo privado. Esto hace de lo común, un verdadero desafío para la sociedad capitalista y patriarcal de nuestros tiempos. Lo común, se ha convertido en aquello que es de todos y de nadie, en lo comúnmente violentado, en lo comúnmente descuidado.

¿Estamos condenados a vivir en la tragedia de los comunes? ¿será el control de la población la solución a esta tragedia?, o, ¿quizás lo es una gestión de lo común centralizada en el estado? En este ensayo se comparte con Leff (2003) que las soluciones a lo que se conoce como crisis ambiental no se encuentran en las lógicas técnicocientíficas lineales y mecanicistas que aún predominan en la ciencia y la educación, y por lo tanto es necesario de un nuevo saber ambiental, que siguiendo a Leff (2003) "rompa la dicotomía sujeto y objeto del conocimiento para reconocer las potencialidades de lo real y para incorporar valores e identidades en el saber" (28). Bajo este pensamiento, los 
comunes dejan de ser objetos a ser gestionados, para convertirse en dones que deben ser cuidados (Laudato si', 2015).

Sumado a esto se comparte la esperanza latinoamericana de que no solo otros mundos son posibles (Escobar, 2018), sino en que ya existen y que muchos otros se están construyendo. Basta con observar la manera como algunos pueblos originarios han logrado convivir y cuidar lo común para darse cuenta que es posible pensar desde otros lugares que reivindican el valor de la diversidad, la otredad, lo sagrado, lo comunal (Leff, 2003; Noguera, 2004; Escobar, 2005), como apuesta a otras maneras de habitar donde florezca la vida (Noguera, 2004).

\section{Urdimbres para tejer otros vínculos con el agua}

\subsection{Pensamiento ambiental, una invitación polifónica}

Una vez entretejidas las críticas sobre las relaciones mercantiles con las que hoy en día se gestiona el agua y puesto sobre la mesa el problema del cuidado de lo común en las sociedades de hoy, se hace necesario esbozar teóricamente el pensamiento ambiental, al que en este trabajo se le ha añadido los términos de complejo-sur, no para ser rimbombante sino para resaltar posturas epistemológicas y teóricas que, aunque hacen parte de la nutrida construcción filosófica del pensamiento ambiental, permiten dar fuerza al sentido de lo que este escrito desea proponer.

Teniendo en cuenta el alcance de este trabajo y sin la intención de caer en reduccionismos, se presentará de manera muy sucinta lo que connota hablar de lo que se ha llamado pensamiento ambiental. Se puede decir entonces, que el pensamiento ambiental representa una invitación emanada de la filosofía ambiental de pensadores de América del sur. Desde este pensamiento se cuestionan los discursos hegemónicos capitalistas, patriarcales y colonizadores, que han buscado controlar la naturaleza y mercantilizarla. En contraste a esto, el pensamiento ambiental está nutrido de diversas voces que en el marco de la pluralidad permiten develar la complejidad de lo ambiental, desde posturas decoloniales y de resistencia.

En Latinoamérica son diversas las propuestas que pueden cobijarse bajo lo que se considera pensamiento ambiental, entre las más destacadas se encuentra lo que se conoce como buen vivir, sin embargo, en términos de escuelas de pensamiento ambiental se destacan la corriente de ecología política en México y la corriente de pensamiento ambiental de Augusto Ángel maya en Colombia. La visión de este trabajo se fundamenta principalmente en esta segunda corriente, que plantea una postura estética del pensamiento ambiental, en donde se abraza la idea de disolver de la dicotomía sujeto/objeto para podernos sentir cuerpos-tierra, y se invita a transitar los caminos del sentir para ese re-encuentro con la tierra que somos (Noguera, 2007).

Es así, como desde la Escuela de Pensamiento Ambiental de Augusto Ángel Maya, en cabeza de Ana Patricia Noguera, se plantea la urgencia de un pensamiento vivo y de la 
vida, un pensamiento que las culturas de la América - Abya-Yala, cuidan en clave de un habitar poético, de un habitar-sur. (Noguera, 2013). En esta dirección hablar de cuidado en clave de una pensamiento ambiental complejo-sur, es hablar de la reivindicación de los saberes ancestrales, populares y de sentido común que han sido desdeñados por la ciencia, con el fin de recuperar el vínculo con lo sagrado, lo diverso como camino y el sentir como forma de conocer.

Antes de continuar con lo que se desarrollará en el siguiente apartado, se hace necesario aclarar que la alusión a lo complejo-sur que se realiza aquí, toma como punto de referencia el pensamiento complejo de Edgar Morín, quien con su propuesta dialógica (Morin - Ciurana - Motta, 2002), permite religar los diversos sentipensares que alrededor del agua, se han suscitado del proceso investigativo que enmarca este trabajo. Sin dejar de lado el sentido amplio de lo complejo, este ensayo en particular se limita a retomar el principio dialógico moriniano para establecer un diálogo de saberes que reconoce la multidimensionalidad del ser y la complejidad del mundo, así mismo, se habla del sur como metáfora y no como lugar geográfico, buscando reconectar lo que existe de afectivo en la vida para comprender el lenguaje de la tierra.

\subsection{El cuidado como forma de habitar}

Tomando el cuidado como principio orientador para repensar la gestión del agua, se emprende un viaje con acompañado de la voz de Leonardo Boff quien permite caminar hacia una comprensión de la complejidad ambiental desde una ética del cuidado, esto es, desde una relación con nosotros mismos, con la otredad y con la tierra basada en una actitud amorosa y protectora (Boff, 2001).

Este andar se inspira en el deseo de buscar caminos, en los cuales el ser humano se reconozca como hijo del gran amor de la vida y reestablezca sus vínculos con el agua, para esto, se sustentan las razones por las cuales se considera que el cuidado representa una actitud fundamental para afrontar los desafíos de la crisis ambiental. Para comenzar esta travesía es fundamental reconocer dos premisas, por un lado que la crisis ambiental es la consecuencia del descuidado y que esta falta de cuidado pone en riesgo la permanencia de la especie humana en el planeta (Boff, 1999); y que nadie se da la vida a sí mismo, si no que la recibe de alguien que la acoge solidariamente y la introduce en la comunidad de los humanos, es por esto que se considera que el cuidado posibilita los sentimientos de solidaridad y compasión necesarios para convivir en armonía (Boff, 2001).

Quizás por el afán de alcanzar las metas del progreso y el desarrollo, la humanidad ha caminado con la mirada puesta en una ilusión que enceguece y no deja ver lo que se está pisando y/o dejando atrás en el camino. Con "la razón" como única luz que alumbra este caminar, los seres humanos han transitado por la vida tras la promesa de riqueza y crecimiento infinito sin detenerse para mirar atrás y sí que menos hacia adelante. Es 
momento de detenerse y preguntarse ¿Cómo es posible habitar poéticamente hacia un florecimiento de la vida?.

Se presenta entonces el cuidado como una forma de habitar el mundo, no se necesita ser un intelectual para saber que algo o alguien se cuida como resultado de un vínculo afectivo, de una relación amorosa, y el amor es el sentimiento que permite el florecimiento de la vida. Basta con pensar en la vida de un bebé recién nacido, para comprender que sin el cuidado del otro, no es posible que sobreviva. Ahora bien, desde su sentido etimológico, la palabra cuidado, proviene del latín cogitare que significa pensar, reflexionar, que a su vez se compone del prefijo co que significa acción conjunta y agitare que alude a poner en movimiento. De esta manera repensar el cuidado del agua en clave del pensamiento ambiental complejo-sur es una invitación a pensar y actuar colectivamente desde una movilización amorosa.

\section{Trama investigativa}

\subsection{Preludio}

Bajo las premisas anteriores, que argumentan la necesidad de repensar lo ambiental desde las nociones de lo común (Ostrom, 2009) lo diverso (Leff, 2003), lo sagrado (Boff, 1999), lo sentido (Noguera, 2004), se adelanta la tesis doctoral que hoy permite la escritura de este ensayo. Este trabajo investigativo se titula «Praxis Pedagógica en clave de pensamiento ambiental complejo-sur» y se desarrolla para la obtención del título de Doctor en Ciencias de la Educación de la Universidad Simón Bolívar de Barranquilla, Colombia. Dicha tesis se desarrolla en el marco articula al proyecto regional titulado "Resiliencia y Gestión de Sistemas Socio-Ecológicos Estratégicos para la Actividad Agrícola, en Escenarios de Variabilidad Climática. Caso de Estudio: Lago Sochagota (Boyacá, Colombia)", en adelante proyecto BIO-293, buscando aportar escenarios pedagógicos que posibiliten una gestión que reconozca la diversidad de intereses y voces en el territorio y democratice el acceso a la información con miras a una toma de decisiones que maximicen el beneficio de cada uno de los actores involucrados en el proyecto.

El proyecto Proyecto BIO-293, toma como área de estudio un sistema socioecológico que abarca el Lago Sochagota junto con los cuerpos de agua dulce y termomineral con los cuales se conecta, sistema asociado a la cuenca alta del río Chicamocha. Dicho sistema socio-ecológico es estratégico para el desarrollo de actividades agrícolas, turísticas, mineras y de consumo humano. Dado que la gestión y operación de este sistema puede comprometer la calidad y la cantidad de agua necesaria para la resiliencia del sistema y por ende su aprovechamiento por parte de todos, este proyecto se propone el diseño y formulación de un modelo de gestión con enfoque regional que permita el aprovechamiento y manejo del recurso. 
Cuidado del agua en clave del pensamiento ambiental complejo-sur

Como puede leerse en el párrafo anterior, se trata de un proyecto de carácter principalmente técnico que busca la "gestión del recurso", sin embargo dada la complejidad de los sistemas socio-ecológicos, la postura epistemológica de este proyecto se plantea bajo un enfoque complejo, el cual permite reconocer y valorar la diversidad de saberes y las relaciones de coproducción a través de las cuales el conocimiento y la toma de decisiones se moldean en sistemas socio-ambientales, no solo como medio para la investigación sino como parte de un proceso social y político (Clark - Lorrae van Kerkhoff - Gallopin, 2016). Con el convencimiento de que la participación de diversos actores y la integración de los conocimientos locales son claves en la construcción de un entendimiento de los sistemas y de sus posibles respuestas a los cambios, el desarrollo de la praxis pedagógica que se propone en la tesis doctoral, en el marco de este proyecto regional, promueve dicha participación, desde un llamado al cuidado del agua.

Ahora, si bien es cierto que continúan existiendo antagonismos entre la ciencia social y la ciencia natural y que el trabajo en equipo interdisciplinar suscita múltiples tensiones, la apuesta de esta andadura investigativa es precisamente esta, conciliar las diferentes posturas teóricas y epistemológicas a través a un diálogo de saberes que reconozca las diferencias. Este reto plantea múltiples interrogantes ¿De qué manera es posible religar los saberes científicos y/o académicos con los saberes populares y ancestrales en busca del cuidado del agua?, ¿Cómo construir puentes de encuentro entre los diferentes intereses (académicos, productivos, institucionales, políticos) que se suscitan en la gestión del Agua?, ¿Cómo superar las limitaciones epistemológicas, disciplinares e institucionales, a favor de generar transformaciones reales en la vida cotidiana hacia el florecimiento de la vida?, ¿Cómo lograr un diálogo de saberes interdisciplinario e intercultural que en clave del pensamiento ambiental complejo-sur que propicie una cultura del cuidado de la vida?

Con los anteriores interrogantes se despliega una propuesta para una praxis pedagógica que a través de un proceso de educación popular logre superar los obstáculos identificados durante esta primera etapa de inmersión y facilite los procesos de diálogo que buscan espacios de apropiación social del conocimiento, concertación de intereses comunes y construcción colectiva de escenarios deseables.

\subsection{Primeras andaduras}

Sin desconocer la experiencia investigativa alrededor del Agua en el Área de Influencia del Lago Sochagota adelantadas en el periodo de 2015 a 2018 por el grupo de investigación que adelanta este trabajo, en donde fue posible algunas acercamientos comunitarios, este escrito se enfoca al trabajo de campo de los últimos meses en el marco de formulación e implementación del Proyecto BIO-293 (octubre 2018 - junio 2019), buscando compartir las primeras reflexiones que surgen de las conversaciones personales y colectivas, que se han suscitado a través de múltiples encuentros informales con los diferentes actores, recorridos de campo por el territorio y la participación constante en escenarios de activismo ambiental y participación ciudadana, en donde haciendo uso de 
la observación participante como técnica para la inmersión en la problemática abordada, se han decantado algunas percepciones sobre las dificultades que se presentan en el territorio alrededor de la gestión del agua.

El diseño metodológico responde a una estrategia de carácter cíclico desde un enfoque complejo que exige una constante observación-planeación-reflexión hacia la comprensión sistémica y holística del problema abordado. La información obtenida en campo, se registra con equipos de grabación de voz, cámara fotográfica y libreta de notas. Se sistematiza con el uso de drive y documentos de Excel. La discusión de los resultados se realiza bajo el reconocimiento previo de las subjetividades e intereses del equipo investigador, con el fin de buscar hacer una devolución de los resultados a los actores, lo más fiel posible a sus propias voces.

\section{Surcando caminos alternos para el cuidado del agua}

Hasta aquí, se ha intentado problematizar la gestión para reconocer la necesidad de otras miradas alrededor del cuidado del agua como bien común (parte 1), se brindaron los referentes teóricos sobre los cuales se sostiene la propuesta de este ensayo (parte 2), y se contextualizó el trabajo investigativo que soporta las propuestas que se pretende aportar en este escrito como el método, técnicas e instrumentos utilizados en la experiencia investigativa (parte 3). En este apartado se comparten los obstáculos epistemológicos que dificultan la gestión del agua que fueron identificados durante el trabajo de campo especialmente en lo relacionado con la toma de decisiones alrededor de este bien común, con el fin de tomarlos como punto de partida para la construcción de un proceso pedagógico que intente superar dichas barreras en clave de un pensamiento ambiental complejo-sur.

A continuación se comparten los resultados en sintonía con el pensamiento ambiental, desde el cual se invita a dar sentido estético, comprendido como afección sensible (Pardo, 1992) al conocimiento, es decir, invita a reconocer que toda forma de conocer es emocional y racional, por esta razón la intención del trabajo que se presenta no es brindar datos objetivos, sino sentipensares subjetivos que han sido validados por los mismos actores a través del diálogo, los cuales permiten tener una percepción de las dinámicas sociales que se tejen alrededor de la gestión del agua en el territorio. 


\subsection{Un devenir de encuentros y desencuentros con almojábana}

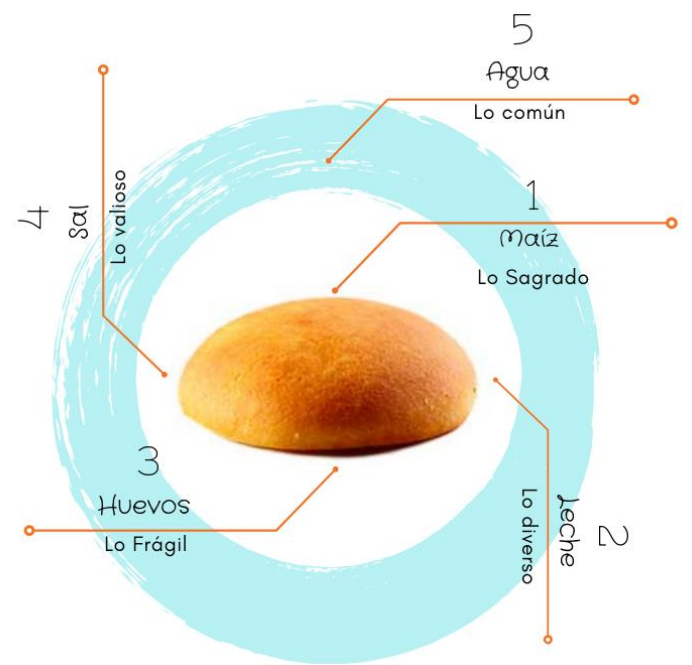

Figura 1. La almojábana es un alimento tradicional desde hace más de 50 años en Paipa, Boyacá (Colombia), por esta razón y por el hecho de que todos los diálogos y encuentros con los actores se han realizado alrededor del compartir una almojábana con masato, se utiliza la imagen de este alimento para presentar una analogía entre los ingredientes con los que se elaboran y las nociones desde la cual se plantea el cuidado del Agua de Paipa en clave del pensamiento ambiental complejo-sur. (Elaboración propia).

\subsection{El maíz y lo sagrado}

Agricultura es una palabra conformada por dos derivaciones latinas "agri” que expresa "arte de cultivar el campo" y "colere", verbo cuya raíz originaria quiere decir "cultivar" y "habitar", de manera que el significado profundo en clave de un pensamiento ambiental complejo del vocablo agricultura es "el arte de cultivar y habitar la tierra" (Giraldo, 2013). Con esta noción de agricultura como referente, se escuchan los sentipensares no solo de agricultores sino de las demás personas con las que se ha interactuado y se identifica que aunque se encuentran expresiones que resaltan la importancia del agua para la obtención de alimento, no se percibe la actividad agrícola como un saber de alto valor para la vida, es decir, el mismo campesino reduce su actividad a una actividad netamente productiva sin relacionarla con una forma de vida que lo define y que le exige la responsabilidad de cultivar vida, y si bien es cierto, que hasta el momento no ha sido posible hacer entrevistas a profundidad que permitan llegar a conclusiones sobre las percepciones de la actividad agrícola en Paipa, si se ha logrado escuchar narrativas que manifiestan inconformidades ante lo que consideran el trabajo duro del campo, la incapacidad para competir en el mercado y la incertidumbre frente a la actual variabilidad climática; en cuanto al Agua, la 
mayoría de población, es decir no solo los agricultores, expresa preocupación con respecto la cantidad de este elemento vital.

Lo anterior permite percibir que estas entre otras muchas dificultades con las que tienen que vivir los agricultores, han generado un desencanto del arte de cultivar y con esto una pérdida del carácter sagrado que tiene no solo el alimento en sí mismo sino el ritual de siembra y cosecha que ha mantenido por generaciones las comunidades campesinas. Lo anterior se afirma desde el compartir con personas que habitan el campo y han decidido dejar de cultivar alimentos o reducir esta actividad al consumo familiar, y que narran con nostalgia que han tenido que buscar trabajo en otras actividades. Ante esto, se identifica un obstáculo epistemológico que se considera importante superar si se quiere trabajar en la resiliencia y la gestión del agua en el sector agrícola, este obstáculo lo podemos denominar pérdida de lo sagrado.

Este obstáculo se ha relacionado con el maíz, por ser este grano considerado el alimento sagrado de Latinoamérica, y esto nos permite hacer alusión al desencanto del arte de cultivar y habitar la tierra, además vale la pena mencionar que este grano es uno los principales cultivos junto con el cultivo de cebolla, frijol y papa (Koenders, 2018) en Paipa. Para explicar la idea de la pérdida de lo sagrado, se hace necesario hablar de la noción de reencantamiento del mundo planteada por Noguera (2004), en donde expresa:
Creo que buscar un reencantamiento del mundo es salir de la visión cientificista de un mundo reducido a una fórmula matemática, a un mundo donde lo sagrado, lo innombrable, lo hermético, aquello de lo cual solo podemos decir que es, se torna en propuesta de sentidos de mundo donde nosotros somos poiesis de ese mundo que es potencia (21).

Según Noguera (2011) renunciar a lo sagrado es renunciar a la naturaleza, porque la naturaleza es asombro y la pérdida de la capacidad de asombro ante el maravilloso acto de producir alimento, es una pérdida de lo sagrado. Reconociendo que el desencanto no solo de agricultores sino de toda la población ante el arte de cultivar, dificulta el trabajo de resiliencia en la gestión del agua, en la medida que genera indiferencia y resignación ante la toma de decisiones relacionadas con este elemento vital. En este trabajo, que como ya se mencionó anteriormente tiene intereses pedagógicos, es necesario reencantar el mundo de la agricultura, para que tanto productores como consumidores tengan curiosidad sobre el elemento que posibilita el alimento, es decir el agua y deseen conocer, participar y trabajar mancomunadamente para hacer del cuidado del agua un arte.

Ahora bien, las múltiples actividades turísticas, ganaderas, agrícolas y mineras presentes en el territorio, hacen que el agua se reconozca como un recurso indispensable para las actividades productivas, pero no como un elemento sacro que posibilita la vida misma, por esto se propone una apuesta pedagógica que intente superar estas barreras epistemológicas que impiden otros vínculos con el agua. Para esto se propone trabajar en la recuperación y reivindicación del saber ancestral como camino de reencuentro con lo 
nuestro, y de eslabón para aceptar con humildad que existen límites del conocimiento (Nicolescu, 1996), y que existen para cuidar el agua, es indispensable dar cabida a los misterios de la vida.

\subsection{La leche y lo diverso}

En los encuentros con los diferentes actores del sistema del Lago Sochagota, se denota una maravillosa diversidad de voces que aunque algunas veces disonantes, enriquecen el diálogo y nutren las propuestas hacia el cuidado del agua, sin embargo las conversaciones con instituciones, agricultores, ganaderos, hoteleros y líderes ambientalistas dan cuenta de dificultades para diálogo en medio de la diversidad, poca capacidad para conciliar los diferentes intereses de los actores involucrados y una alta predisposición a los proyectos institucionales y de manera especial a los estudios académicos, ya que manifiestan que se han hecho muchas investigaciones en la zona y no se evidencian transformaciones reales en el territorio.

El encuentro en la diversidad se convierte en una apuesta fundamental para el cuidado de este bien común, por eso es necesario abrirse a la idea de que no existe una única forma de cuidar, sino que por el contrario es la suma de muchas formas de cuidado las que permiten una efectiva participación e inclusión de todos los saberes que comparten esta empresa. Cuidar la diversidad desde la diversidad es comprender un mundo plural, donde se da cabida a todo ser vivo, sin importar su valor en términos de utilidad, sino reconociendo el valor que cada cosa (viva o no viva) tiene en sí misma.

De esta manera utilizando el don de la leche para generar diversidad de productos (yogures, kumis, cuajadas, quesos, mantequilla) se hace alusión a la necesidad de reconocer el valor de lo diverso para el cuidado de lo común. En este sentido, surgen apuestas pedagógicas orientadas a superar las dificultades epistemológicas de los diferentes actores del territorio (incluyendo el equipo de investigadores) que impiden un reconocimiento del otro desde la comprensión de múltiples realidades. La escucha del otro en su diversidad se convierte entonces, en un reto a alcanzar en el marco del proceso pedagógico que se espera desarrollar para lograr en este trabajo, ya que la diversidad, es el reflejo mismo de la vida que nos invita a abrazar la diferencia y la pluralidad, como aspectos claves para la resiliencia de los sistemas socio-ecológicos.

\subsection{Los huevos y lo frágil}

Compartiendo la convicción de Maya (2015) de que "las formas de organización social están íntimamente vinculadas a la transformación tecnológica de los ecosistemas”(8), y que "las racionalidades sociales, económicas y políticas influyen en el mejoramiento o deterioro de los sistemas naturales" (9), en este apartado se abordan algunas percepciones alrededor de aspectos culturales que se identifican como barreras al cuidado de lo común, vale la pena aclara que dichas percepciones son el resultado de la experiencia subjetiva 
del autor de este escrito, quien no solo mediante los encuentros comunitarios realizados en el marco del proyecto BIO-293 sino durante el trabajo de más de 5 años de activismo ambiental en el territorio ha ido decantando y que resultan claves para abordar la resiliencia del sistema socio-ecológico estudiado, comprendida dicha resiliencia como la capacidad de un sistema natural y cultural para absorber, adaptarse, transformarse y autoorganizarse ante escenarios de cambio e incertidumbre (Sánchez, 2011).

La necesidad de abordar los aspectos culturales en el marco de lo ambiental, surge de la comprensión de "lo ambiental" como el resultado de la relación cultura-ecosistema (Maya, 2005) y de la noción de la fragilidad ambiental de la cultura (Maya, 2015), referentes teóricos que permiten fundamentar la interdependencia entre el ambiente natural y la construcción de la cultura de una sociedad. Bajo esta perspectiva, es imposible dejar de lado los aspectos culturales que en el marco de este proyecto permiten reflexionar sobre el reconocimiento de la interdependencia y de la fragilidad de la resiliencia cultural frente al medio, con el fin de identificar estrategias de adaptación y autoorganización que permitan el cuidado de lo común.

Para abordar lo anterior, es necesario hacer alusión al fenómeno de homogeneización de la cultura en términos del sistema de acumulación promovido por el capitalismo, ya que no se puede negar que una de las consecuencias de este sistema capitalista, es la implantación de una cultura individualista basada en principios de autonomía y libertad que en la mayoría de los casos impide la construcción comunidades interconectadas y solidarias. Planeando dicha homogeneización de la cultura como un problema ambiental, se considera que la población de Paipa no escapa a este fenómeno y se puede percibir la manera como no se reconocen las interdependencias entre las personas como tal y de estas con el ecosistema que habitan. Esta apreciación se deriva también, de los intereses personales que se manifiestan en los distintos encuentros, en donde se expresa peticiones que buscan compensaciones personales o financiamientos de proyectos individuales.

En atención a la resistencia a una cultura de acumulación de riqueza e individualismo, se plantea la necesidad de trabajar en procesos pedagógicos que resignifique la relación cultura-ecosistema desde el reconocimiento de la interdependencia no solo entre actores sino entre cada actor con el ecosistema que habita. Esto implica un distanciamiento del antropocentrismo fuerte con el que la humanidad se ha relacionado con el ecosistema para dar cabida a una relación ética basada en la reciprocidad.

Es así como los huevos representando la fragilidad ambiental de la cultura, deben ser cuidados con mucha delicadeza, porque dicha fragilidad implica que cada pequeña decisión tecnológica, económica y política genera adaptaciones tanto del ecosistema como de la cultura que pueden ser favorables y/o desfavorables para la resiliencia de los sistemas socio-ecológicos. Es por esta razón, que la toma de decisiones se convierte en una responsabilidad ante dicha fragilidad, o mejor dicho una responsabilidad ante el alcance de un nivel de resiliencia que permita tanto a la cultura como al ecosistema afrontar la incertidumbre con creatividad social. 
Cuidado del agua en clave del pensamiento ambiental complejo-sur

\subsection{La sal y lo valioso}

La sal representa en este caso la necesidad de dar valor moral y espiritual a los bienes comunes, pero también la necesidad ya sea de encontrar alternativas sustentables de generación de ingresos o formas de mejorar los procesos productivos existentes en el marco de la sostenibilidad. En el territorio se evidencia un reconocimiento hacia los daños que las diferentes actividades productivas ocasionan al ambiente, sin embargo, los procesos de cambio que se requieren para tecnificar y mejorar dichas prácticas se ven lejanas no solo por la falta de fortalecimiento de capacidades de las personas sino por el temor que se tiene al cambio en sí mismo. Es necesario que a través de una pedagogía se promueva la creatividad social y el emprendimiento encaminada a una generación de valor que brinde la posibilidad de tener una vida digna basada en el bien común.

También, vale la pena compartir que durante el desarrollo de la investigación se propuso la realización de una ruta pedagógica que fue convocada bajo el nombre "Agua de Luna para la Sal de la tierra”, en la cual participaron investigadores, estudiantes universitarios y líderes sociales de Paipa, este caminar compartido, permitió el diálogo sobre algunos aspectos históricos alrededor de la sal en Paipa, la cual deviene de la presencia de aguas termominerales, que a su vez se han convertido en el principal atractivo turístico de Paipa por sus propiedades medicinales. Alrededor de la sal, fue posible poner de manifiesto el valor no solo de los minerales y de sus poderes sanatorios sino el valor de su cultura y sus tradiciones.

\subsection{E1 Agua y lo común}

Para terminar, se presenta el Agua como aquello que todo lo conecta. Este apartado se limita a dejar una gran desolación de haber perdido el lenguaje para lo común, de haber olvidado lo comunal como forma de vida, y resaltar con tristeza la manera como la narrativas de los diferentes actores, transitan entre la identificación de culpables de los problema del agua y las solicitudes de asignar un responsable del Lago Sochagota, casi en un intento por buscar un dueño que se haga cargo y asuma toda responsabilidad sobre este cuerpo de agua. Desde este hallazgo, se reconoce la urgencia de rescatar la vida comunitaria, siendo este el mayor reto para poder transitar los caminos del cuidado. Siendo largo el camino para el reencuentro, se espera que el andar compartido sirva de escenario pedagógico para aprender, desaprender, y reaprender a cuidar lo común.

\section{Conclusión}

Ya se quisiera brindar respuestas o soluciones a la problemática aquí plasmada, pero infortunadamente, solo se pueden dejar más preguntas, ¿Cómo cuidarse y cuidar-nos en un mundo liderado por los intereses del mercado?, ¿Qué hacer para lograr restablecer las relaciones de confianza necesarias para actuar con responsabilidad colectiva?, ¿Cómo 
cuidar los bienes comunes desde las diferentes formas de concebir el cuidado y desde los diferentes intereses que se tiene sobre el bien común?, ¿Cómo evitar que el cuidado se convierta un mecanismo de control?, ¿Quién decide cómo cuidar?, ¿Cuál es el conocimiento sobre el cual se toman decisiones para cuidar un bien común?.

Bajo este panorama, se llega a la conclusión que, para cuidar, no solo es necesario abrir nuevos caminos sino aprender a caminarlos de nuevas maneras, y para eso es vital una pedagogía del vínculo, que permita reconocerse como parte de un todo interconectado, una pedagogía que rompa con la cultura de individualismo para generar valores comunales y que coadyuve a una creatividad social en donde todo tipo de saber sea valioso para repensar el mundo que se habita. En este trabajo se ha intentado dejar algunas puntadas sobre los obstáculos epistemológicos alrededor del cuidado de lo común con el fin de brindar herramientas pedagógicas que coadyuven a la construcción colectiva de resiliencia en el marco de la diversidad y la reciprocidad.

Es necesario seguir tejiendo con diversas manos esta colcha de retazos, porque lo cierto es que, algo que suena tan sencillo como decir "cuidemos el agua", se convierte en un gran desafío, en el cual, investigadores, agricultores, instituciones y movimientos sociales, deben buscar de manera creativa esas alternativas de cuidado, que con el mínimo grado de dominación posible permitan que todos puedan disfrutar de este elemento vital. Quizás no sea posible saber con certeza si en ese intento de cuidar realmente estamos cuidando, pero es nuestra responsabilidad intentarlo.

\section{Referencias bibliográficas}

Apipalakul, C. - Wirojangud, W. - Ngang, T. K. (2015). Development of community participation on water resource conflict management. Procedia-Social and Behavioral Sciences, 186. 325-330.

Boff, L. (2001). Ética planetaria desde el gran sur. Madrid: Trotta.

Boff, L. (1999). Saber cuidar. Petrópolis: Vozes, 32.

Clark, W. C. - van Kerkhoff, L. - Lebel, L., - Gallopin, G. C. (2016). Crafting usable knowledge for sustainable development. Proceedings of the National Academy of Sciences, 113/17. 4570-4578.

Escobar, A. (2018). Otro posible es posible: Caminando hacia las transiciones desde Abya Yala/Afro/Latino-América. Bogotá: Desde Abajo.

Escobar, A. (2005). Más allá del tercer mundo: globalización y diferencia. Bogotá: Instituto Colombiano de Antropología e Historia, Universidad del Cauca.

Koenders, L. (2018). Irrigation management in Colombia. Farmers and the water users association management of salt water in the Chicamocha irrigation system, Department Boyaca, Colombia (Tesis de Maestría). Netherlands: Wageningen University.

Leff, E. (2003). Complejidad Ambiental. Siglo XXI Editores. 
Cuidado del agua en clave del pensamiento ambiental complejo-sur

Maya, A. (2015). La Fragilidad ambiental de la cultura. Historia y medio ambiente. Segunda edición. Publicación en línea: www.augustoangelmaya.com.

Maya, A. A. (1996). El reto de la vida. Ecosistema y cultura. Una introducción al estudio del medio ambiente. Bogotá: Ecofondo.

Morin, E. - Ciurana, E. R. - Motta, R. D. (2002). Educar en la era planetaria: el pensamiento complejo como método de aprendizaje en el errory la incertidumbre humana. Universidad de Valladolid.

Nicolescu, B. (1996). La transdisciplinariedad. Mónaco: Du Rocher. Asequible en: https:/ / amigosinterdis.files.wordpress.com/2019/01/la-transdisciplinariedad_-basarabnicolescu.pdf, fecha de consulta: 03-08-2020.

Noguera, A. P. (2011). Las potencias del olvido en el habitar ambiental. Primer Seminario Latinoamericano sobre Pensamiento Ambiental y Hábitat, Movimiento Allegro: El Territorio, Experiencia Reflexiva en Hábitat. Medellín.

Noguera, A. P. (2010). Cuerpo-Tierra. Ethos ambiental en clave de la lengua de la Tierra. Revista Sustentabilidad(es), 2. Asequible en: http:/ /www.sustentabilidades.usach.cl/numero -2-ano-1-julio-2010\#overlay-context=node/29/edit, fecha de consulta: 03-08-2020.

Noguera, A. P. (2007). Hojas de Sol en la Victoria Regia: Emergencias de un pensamiento ambiental alternativo en América Latina. Colombia: Universidad Nacional.

Noguera, A.P. (2004). El Reencantamiento del Mundo. Universidad Nacional de Colombia.

Ostrom, E. (2009). El gobierno de los bienes comunes. La evolución de las instituciones de acción colectiva. México: FCE; UNAM; CRIM; IIS.

Papa Francisco. (2015). Laudato si’: Carta encíclica sobre el cuidado de la casa común. Palabra. Real Academia Española. (2001). Disquisición. En Diccionario de la lengua española (22.a ed.). Asequible en: https://dle.rae.es/?id=JAQijnd, fecha de consulta: 03-08-2020. Rodríguez Brandao, C. - Fals Borda, O. (1986). Investigación participativa. Montevideo: Instituto Del Hombre/Ediciones de la Banda Oriental.

Sánchez, A. (2011). Conceptos básicos de gestión ambiental y desarrollo sustentable. México: Secretaría de Medio Ambiente y Recursos Naturales, Instituto Nacional de Ecología, Asociación para el desarrollo integral de la región de Misantla, S y G editores.

Tangencial, T. (2002). Manifiesto por la vida por una ética para la sustentabilidad. Ambiente \& Sociedade, 5(10). 1-14.

Thompson, M. A. - Owen, S. - Lindsay, J. M. - Leonard, G. S. - Cronin, S. J. (2017). Scientist and stakeholder perspectives of transdisciplinary research: Early attitudes, expectations, and tensions. Environmental Science \& Policy, 74. 30-39.

UNESCO, (2019). Informe Mundial de las Naciones Unidas sobre el Desarrollo de los Recursos Hidricos. Programa Mundial de Evaluación de los Recursos Hidricos WW AP. París: UNESCO. 